\title{
Article
}

\section{Imprecise system reliability and component importance based on survival signature}

Feng, Geng, Patelli, Edoardo, Beer, Michael and Coolen, Frank P.A.

Available at http://clok.uclan.ac.uk/25623/

Feng, Geng ORCID: 0000-0002-4705-3252, Patelli, Edoardo, Beer, Michael and Coolen, Frank P.A. (2016) Imprecise system reliability and component importance based on survival signature. Reliability Engineering \& System Safety, 150 . pp. 116-125. ISSN 0951-8320

It is advisable to refer to the publisher's version if you intend to cite from the work. http://dx.doi.org/10.1016/j.ress.2016.01.019

For more information about UCLan's research in this area go to http://www.uclan.ac.uk/researchgroups/ and search for <name of research Group>.

For information about Research generally at UCLan please go to http://www.uclan.ac.uk/research/

All outputs in CLoK are protected by Intellectual Property Rights law, including Copyright law. Copyright, IPR and Moral Rights for the works on this site are retained by the individual authors and/or other copyright owners. Terms and conditions for use of this material are defined in the policies page.

\section{CLoK}

Central Lancashire online Knowledge www.clok.uclan.ac.uk

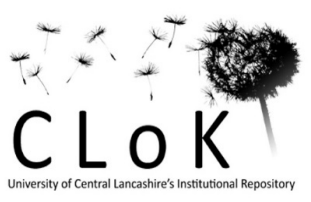




\section{Author's Accepted Manuscript}

Imprecise System Reliability and Component Importance Based on Survival Signature

Geng Feng, Edoardo Patelli, Michael Beer, Frank P.A. Coolen

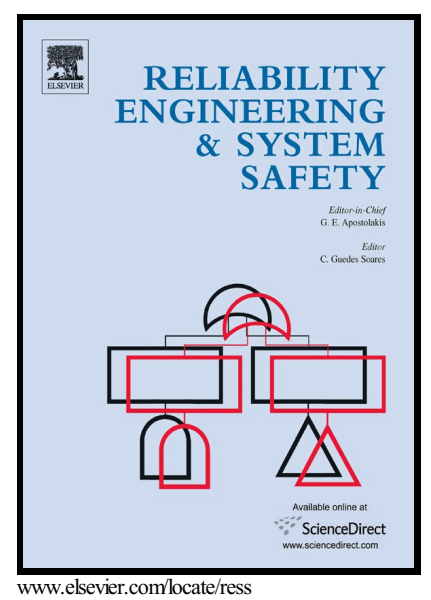

PII: $\quad$ S0951-8320(16)00028-4

DOI: $\quad$ http://dx.doi.org/10.1016/j.ress.2016.01.019

Reference: RESS5493

To appear in: Reliability Engineering and System Safety

Received date: 11 June 2015

Revised date: 26 January 2016

Accepted date: 27 January 2016

Cite this article as: Geng Feng, Edoardo Patelli, Michael Beer and Frank P.A Coolen, Imprecise System Reliability and Component Importance Based o. Survival Signature, Reliability Engineering and System Safety http://dx.doi.org/10.1016/j.ress.2016.01.019

This is a PDF file of an unedited manuscript that has been accepted fo publication. As a service to our customers we are providing this early version o the manuscript. The manuscript will undergo copyediting, typesetting, an review of the resulting galley proof before it is published in its final citable form Please note that during the production process errors may be discovered whic could affect the content, and all legal disclaimers that apply to the journal pertain 


\title{
Imprecise System Reliability and Component Importance Based on Survival Signature
}

\author{
Geng Feng, Edoardo Patelli, Michael Beer \\ Institute for Risk and Uncertainty, School of Engineering, University of Liverpool, \\ Liverpool, United Kingdom \\ Frank P.A. Coolen \\ Department of Mathematical Sciences, Durham University, Durham, United Kingdom
}

\begin{abstract}
The concept of the survival signature has recently attracted increasing attention for performing reliability analysis on systems with multiple types of components. It opens a new pathway for a structured approach with high computational efficiency based on a complete probabilistic description of the system. In practical applications, however, some of the parameters of the system might not be defined completely due to limited data, which implies the need to take imprecisions of component specifications into account. This paper presents a methodology to include explicitly the imprecision, which leads to upper and lower bounds of the survival function of the system. In addition, the approach introduces novel and efficient component importance measures. By implementing relative importance index of each component without or with imprecision, the most critical component in the system can be identified depending on the service time of the system. Simulation method based on survival signature is introduced to deal with imprecision within components, which is precise and efficient. Numerical example is presented to show the applicability of the approach for systems.
\end{abstract}

Keywords: Imprecision; survival signature; system reliability; component importance; sensitivity analysis.

\footnotetext{
* Corresponding author

Email address: edoardo.patelli@liverpool.ac.uk (Edoardo Patelli)
} 


\section{INTRODUCTION}

Networked systems are a series of components interconnected by communication paths. The analysis of these systems becomes more and more important as they are the backbone of our societies. Examples include the Internet, social 5 networks of individuals or businesses, transportation network, power plant system, metabolic networks, and many others. Since the breakdown of a system may cause catastrophic effects, it is essential to be able to assess the reliability and availability of these systems. As an intrinsic feature, practical systems involve uncertainties to a significant extent. Since the reliability and performance of systems are directly affected by uncertainties, a quantitative assessment of uncertainty is widely recognized as an important task in practical engineering [1]. The obvious pathway to a realistic and powerful analysis of systems is a probabilistic approach. In practical cases there are two specific challenges that need to be addressed to obtain realistic results. First, the complexity of the system needs to be reflected in the numerical model. This goes far beyond a model based on a set of components with simple connections between them. For instance, there may be several different types of components in the same system. This variety together with the large size of real-life systems complicates the propagation of the uncertainty from the various different component types with their different performance and uncertainty characteristics to the system performance for the prediction of the system lifetime and reliability. Second, the available information for the quantitative specification of the uncertainties associated with the components is often limited and appears as incomplete information, limited sampling data, ignorance, measurement errors and so forth.

25 The present work contributes towards a solution to these challenges.

The proposed approach is based on the survival signature, which is associated with a survival analysis [2] of systems. Survival analysis has important applications in biology, medicine, insurance, reliability engineering, demography, sociology, economics, etc. In engineering, survival analysis is typically 
referred to as reliability analysis, and the survival function is then called reliability function. This survival function or reliability function quantifies the survival probability of a system at a certain point in time. In this context, the concept of the system signature [3] has been recognized as an important tool to quantify the reliability of systems that consist of exchangeable components.

35 The main advantage of the system signature is its capability to separate the structure of the system from the probabilistic model used to describe the random failure of the system components. Recent advancements using the concept of system signature are reported in [4]. However the use of the system signature is associated with the assumption that all components in the system are of the same type. This is a major limitation since real systems are generally formed by more than one component type so that those systems cannot be analysed with the system signature [5].

In order to overcome the limitations of the system signature, Coolen and Coolen-Maturi [5] proposed the survival signature as improved concept, which 45 does not rely any more on the restriction to one component type. Specifically, the characteristics of the components do not need to be independently and identically distributed (iid). In the case of a single component type, the survival signature is closely related to the system signature. Recent developments have opened up a pathway to perform a survival analysis using the concept of survival so signature even for relatively complex systems. Coolen et al. have shown how the survival signature can be derived from the signatures of two subsystems in both series and parallel configuration [6], and they developed a non-parametric predictive inference scheme for system reliability using the survival signature [5]. Aslett et al. [7] presented the use of the survival signature for systems reliability quantification from a Bayesian perspective.

In many cases, uncertainties cannot be quantified precisely since they are characterized by incomplete information, limited sampling data, ignorance, measurement errors and so on. Thus, a thorough and realistic quantitative assessment of the uncertainties is quite important. Moreover, it is essential to know which component with uncertainties has the biggest influence degree to the 
whole system.

Component importance measure allows to quantify the importance of system components and identify the most "critical" component. It is a useful tool to find weaknesses in systems and to prioritize reliability improvement activities.

${ }_{65}$ Birnbaum [8] proposed a measure to find the reliability importance of a component in 1969, which is obtained by partial differentiation of the system reliability with respect to the given component reliability. An improvement or decline in reliability of the component with the highest importance will cause the greatest increase or decrease in system reliability. Several other importance measures have been introduced [9]. Improvement potential, risk achievement worth, risk reduction worth, criticality importance and Fussell-Vesely's measure were all reviewed in Ref. [10] [11] [12] [13]. To conduct reliability importance of components in a complex system, Wang et al. [14] introduced and presented failure criticality index, restore criticality index and operational criticality index. Zio et al. [15] [16] presented generalized importance measures based on Monte Carlo simulation. The component importance measures can determine wiich components are more important to the system, which may suggest the most efficient way to prevent system fails.

Some of the importance measures can be computed through analytical methso ods, but limited to systems with few components. Traditional simulation methods provide no easy way to compute component importance [14]. In addition, in case with imprecision in the component failure, the simulation approaches become intractable.

In this paper, a novel reliability approach and component importance mea${ }_{85}$ sure based on survival signature is proposed to analyse systems with multiple types of components. The proposed approach allows to include explicitly imprecision and vagueness in the characterization of the uncertainties of system components. The imprecision characterizes indeterminacy in the specification of the probabilistic model. That is, an entire set of plausible probabilistic models 90 is specified using set-values (herein, interval-valued) descriptors for the description of the probabilistic model. The cardinality of the set-valued descriptors 
reflects the magnitude of imprecision and, hence, the amount and quality of information that would be needed in order to specify a single probabilistic model with a sufficient confidence. In real cases the amount and quality of information to specify a probabilistic model can be limited to such an extent that the associated magnitude of imprecision makes the entire analysis meaningless. In such cases it is essential to identify those contributions to the imprecision, which influence the results most strongly. Once these are known, targeted measures and investments can be defined in order to reduce the imprecision to enable a meaningful survival analysis. For this purpose, a component importance measure is implemented to identify the most "critical" component of the system taking into account the imprecision in their characterization. Specifically, new component importance measure is introduced as the relative importance index $(R I)$. Through simulation method based on survival signature, upper and lower bounds of survival function of the system or relative importance index can be got efficiently. On this basis, the survival function of system and the importance degree of components can be quantified. The proposed approaches of the improved survival signature are demonstrated by some examples.

\section{SURVIVAL SIGNATURE AND SURVIVAL FUNCTION}

Suppose there is one system formed by $m$ components. Let the state vector of components be $\underline{x}=\left(x_{1}, x_{2}, \ldots, x_{m}\right) \in\{0,1\}^{m}$ with $x_{i}=1$ if the $i$ th component is in working state and $x_{i}=0$ if not. $\phi=\phi(\underline{x}):\{0,1\}^{m} \rightarrow\{0,1\}$ defines the system structure function, i.e., the system status based on all possible $\underline{x} . \phi$ is 1 if the system functions for state vector $\underline{x}$ and 0 if not.

Now consider a system with $K \geq 2$ types of $m$ components, with $m_{k}$ indicating the number of components of each type and $\sum_{k=1}^{K} m_{k}=m$. It is assumed that the failure times of the same component type are independently and identically distributed (iid) or exchangeable. The components of the same type can be grouped together because of the random ordering of the components in the 120 state vector, which leads to a state vector can be written as $\underline{x}=\left(\underline{x}^{1}, \underline{x}^{2}, \ldots, \underline{x}^{K}\right)$, 
with $\underline{x}^{k}=\left(x_{1}^{k}, x_{2}^{k}, \ldots, x_{m_{k}}^{k}\right)$ representing the states of the components of type $k$. Coolen et al. [6] introduced the survival signature for such a system, denoted by $\Phi\left(l_{1}, l_{2}, \ldots, l_{K}\right)$, with $l_{k}=0,1, \ldots, m_{k}$ for $k=1,2, \ldots, K$, which is defined to be the probability that the system functions given that $l_{k}$ of its $m_{k}$ components of type $k$ work, for each $k \in\{1,2, \ldots, K\}$. There are $\left(\begin{array}{c}m_{k} \\ l_{k}\end{array}\right)$ state vectors $\underline{x}^{k}$ with precisely $l_{k}$ components $x_{i}^{k}$ equal to 1 , so with $\sum_{i=1}^{m_{k}} x_{i}^{k}=l_{k}(k=1,2, \ldots, K)$, and $S_{l_{1}, l_{2}, \ldots, l_{K}}$ denote the set of all state vectors for the whole system.

Assume that the random failure times of components of the different types are fully independent, and in addition the components are exchangeable within the same component types, the survival signature can be rewritten as:

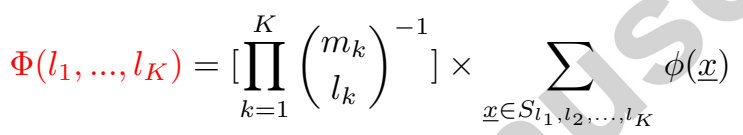

$C_{k}(t) \in\left\{0,1, \ldots, m_{k}\right\}$ denotes the number of $k$ components working at time $t$. Assume that the components of the same type have a known CDF, $F_{k}(t)$ for type $k$. Moreover, the failure times of different component types are assumed independent, then:

$$
P\left(\bigcap_{k=1}^{K}\left\{C_{k}(t)=l_{k}\right\}\right)=\prod_{k=1}^{K} P\left(C_{k}(t)=l_{k}\right)=\prod_{k=1}^{K}\left(\begin{array}{c}
m_{k} \\
l_{k}
\end{array}\right)\left[F_{k}(t)\right]^{m_{k}-l_{k}}\left[1-F_{k}(t)\right]^{l_{k}}
$$

Hence, the survival function of the system with $K$ types of components becomes:

$$
P\left(T_{s}>t\right)=\sum_{l_{1}=0}^{m_{1}} \ldots \sum_{l_{K}=0}^{m_{K}} \Phi\left(l_{1}, \ldots, l_{K}\right) P\left(\bigcap_{k=1}^{K}\left\{C_{k}(t)=l_{k}\right\}\right)
$$

It is obvious from Equation 3 that the survival signature can separate the structure of the system from the failure time distribution of its components, which is the main advantage of the system signature. What is more, the survival signature only need to be calculated once for any system, which is similar to the system signature for systems with only single type of components. It is easily seen that survival signature is closely related with system signature. For a 
special case of a system with only one type $(K=1)$ of components, the survival signature and the Samaniego's signature [3] are directly linked to each other through a simple equation, however, the latter cannot be easily generalized for systems with multiple types $(K \geq 2)$ of components [5].

This implies that all attractive properties of the system signature also hold for the method using the survival signature, also the survival signature is easy to apply for systems with multiple types of components, and one could argue it is much easier to interpret than the system signature.

\section{GENERALIZED PROBABILISTIC DESCRIPTION OF THE FAIL- URE TIMES OF COMPONENTS}

\subsection{Introduction of Probability Box}

As stated in the previous section, the probability of the failure of each component is described by the CDF, $F_{k}(t)$. However, it is not always possible to fully characterize the probabilistic behaviour of components due to ignorance or incomplete knowledge. This lack of knowledge comes from many sources: in-adequate understanding of the underlying processes, imprecise evaluation of the related characteristics, or incomplete knowledge of the phenomena. These problems can be tackled by resorting to generalized probabilistic methods, such as imprecise probabilities, see e.g. [17] [18] [19] [20]. The main problem of generalized probabilistic methods is the computational cost associated with their evaluation. In fact, these approaches required multiple probabilistic model evaluations, and often use global optimization procedures [21]. Efficient numerical methods have been developed and made available in powerful toolboxes such as OpenCossan software [22] [23]. Recently, Coolen et al. have combined nonparametric predictive inference method with survival signature to analyse system reliability [24].

The generalized probabilistic model makes the uncertainty quantification a rather challenging task in terms of computational cost, and the challenge comes mainly from computing the lower and upper bounds of the quantities of interest. 
Let $\underline{F}$ and $\bar{F}$ be non-decreasing functions mapping the real line $\Re$ into $[0,1]$ and $\underline{F}(x) \leq \bar{F}(x)$ for all $x \in \Re$. Let $[\underline{F}, \bar{F}]$ denote a set of the non-decreasing functions $F$ on the real line such that $\underline{F}(x) \leq F(x) \leq \bar{F}(x)$. When the functions $175 \underline{F}$ and $\bar{F}$ circumscribe an imprecisely known probability distribution, $[\underline{F}, \bar{F}]$ is called a "probability box" or "p-box" [25]. Using the framework of imprecise probabilities in form of a p-box (see [26] [27]), the lower and upper CDF for the failure times of components of type $k$ are denoted by $\underline{F}_{k}(t)$ and $\bar{F}_{k}(t)$, respectively. The lower and upper $\mathrm{CDF}$ bounds can be obtained by calculating the range of all distributions that have parameters within some intervals. For some distribution families, only two CDFs need to be computed to enclose the p-box. For most distribution families, however, four or more crossing CDFs need to be computed to define a p-box, see [28]. As an example, Fig. 1 depicts a free p-box whose bounds arise from a lognormal distribution with parameters intervals $\alpha=[\underline{0.5}, \overline{0.6}]$ and $\beta=[\underline{0.05}, \overline{0.1}]$.

\subsection{Analytical Method to Deal with Imprecision within Components Failure} Times

Lower and upper bound of the survival function for a system consisting of multiple types of components can be calculated analytically based on Coolens works for nonparametric predictive inference in [24]. As $C_{k}(t)$ denotes the number of $k$ components working at time $t$, and it is assumed that the components can not be repaired or replaced. The lower survival function is:

$$
\underline{S}_{T_{S}}(t)=\underline{P}\left(T_{S}>t\right)=\sum_{l_{1}=0}^{m_{1}} \ldots \sum_{l_{K}=0}^{m_{K}} \Phi\left(l_{1}, \ldots, l_{K}\right) \prod_{k=1}^{K} \bar{D}\left(C_{k}(t)=l_{k}\right)
$$

where

$$
\bar{D}\left(C_{k}(t)=l_{k}\right)=\bar{P}\left(C_{k}(t) \leq l_{k}\right)-\bar{P}\left(C_{k}(t) \leq l_{k}-1\right)
$$




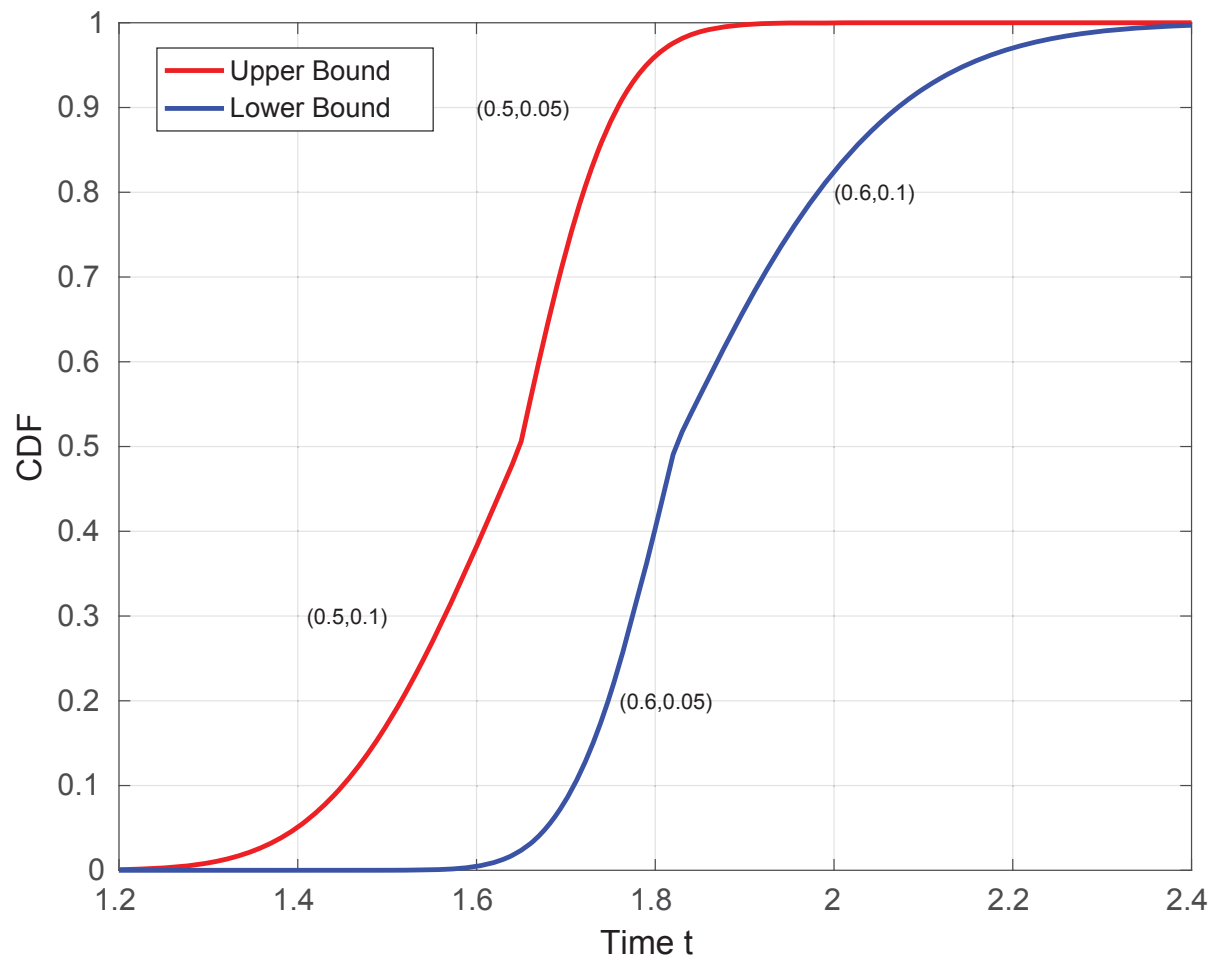

Figure 1: A distributional free p-box whose bounds arise from a lognormal distribution with parameters intervals $\alpha=[\underline{0.5}, \overline{0.6}]$ and $\beta=[\underline{0.05}, \overline{0.1}]$.

While the corresponding upper bound of the survival function is:

$$
\bar{S}_{T_{S}}(t)=\bar{P}\left(T_{S}>t\right)=\sum_{l_{1}=0}^{m_{1}} \ldots \sum_{l_{k}=0}^{m_{K}} \Phi\left(l_{1}, \ldots, l_{K}\right) \prod_{k=1}^{K} \underline{D}\left(C_{k}(t)=l_{k}\right)
$$

\section{where}

$$
\underline{D}\left(C_{k}(t)=l_{k}\right)=\underline{P}\left(C_{k}(t) \leq l_{k}\right)-\underline{P}\left(C_{k}(t) \leq l_{k}-1\right)
$$

For a system with $m$ components in one type, $C_{t}$ is represented to binomial distribution, with $C_{t} \sim \operatorname{Binomial}(m, 1-F(t))$. According to stochastic dominance theory [29], $C_{t}$ increases as $(1-F(t))$ increases.

For parametric distribution, the $\mathrm{CDF}$ of components failure time can be 200 expressed by $F(t \mid \theta)$, with $\theta \in \Theta$ (e.g. parameter $\theta \in[\underline{\theta}, \bar{\theta}])$. Therefore, there 
will be a $\underline{\theta} \in \Theta$ leading to $F(t \mid \underline{\theta})=\underline{F}(t)$ and a $\bar{\theta} \in \Theta$ leading to $F(t \mid \bar{\theta})=\bar{F}(t)$, which holds for all $t$.

Here, taking an exponential distribution with parameter $\lambda \in\left[\lambda_{1}, \lambda_{2}\right]$ as an example. It is known that $\underline{F}(t)=F\left(t \mid \lambda_{1}\right)=1-e^{-\lambda_{1} t}$ and $\bar{F}(t)=F\left(t \mid \lambda_{2}\right)=$ $1-e^{-\lambda_{2} t}$. $C_{t}$ increases as $(1-F(t))$ increases, so $\underline{P}\left(C_{t} \leq l\right)=\sum_{u=0}^{l}\left(\begin{array}{c}m \\ u\end{array}\right)(1-$ $\left.e^{-\lambda_{2} t}\right)^{m-u}\left(e^{-\lambda_{2} t}\right)^{u}$ and $\bar{P}\left(C_{t} \leq l\right)=\sum_{u=0}^{l}\left(\begin{array}{c}m \\ u\end{array}\right)\left(1-e^{-\lambda_{1} t}\right)^{m-u}\left(e^{-\lambda_{1} t}\right)^{u}$.

For a system with one type of components, the lower bound of the survival function for the system at time $t$ becomes:

$$
\underline{S}_{T_{S}}(t)=\underline{P}\left(T_{S}>t\right)=\sum_{l=0}^{m} \Phi(l)\left(\begin{array}{c}
m \\
l
\end{array}\right)\left(1-e^{-\lambda_{1} t}\right)^{m-l}\left(e^{-\lambda_{1} t}\right)^{l}
$$

and the corresponding upper bound of the survival function becomes:

$$
\bar{S}_{T_{S}}(t)=\bar{P}\left(T_{S}>t\right)=\sum_{l=0}^{m} \Phi(l)\left(\begin{array}{c}
m \\
l
\end{array}\right)\left(1-e^{-\lambda_{2} t}\right)^{m-l}\left(e^{-\lambda_{2} t}\right)^{l}
$$

210 $\lambda^{k} \in\left[\lambda_{1}^{k}, \lambda_{2}^{k}\right]$, the lower bound of the survival function for the system at time $t$ is:

$\underline{S}_{T_{S}}(t)=\underline{P}\left(T_{S}>t\right)=\sum_{l_{1}=0}^{m_{1}} \ldots \sum_{l_{k}=0}^{m_{K}} \Phi\left(l_{1}, \ldots, l_{K}\right) \prod_{k=1}^{K}\left(\begin{array}{c}m_{k} \\ l_{K}\end{array}\right)\left[1-e^{-\lambda_{1}^{k} t}\right]^{m_{k}-l_{k}}\left[e^{-\lambda_{1}^{k} t}\right]^{l_{k}}$

The corresponding upper bound of the survival function becomes:

$\bar{S}_{T_{S}}(t)=\bar{P}\left(T_{S}>t\right)=\sum_{l_{1}=0}^{m_{1}} \ldots \sum_{l_{k}=0}^{m_{K}} \Phi\left(l_{1}, \ldots, l_{K}\right) \prod_{k=1}^{K}\left(\begin{array}{c}m_{k} \\ l_{K}\end{array}\right)\left[1-e^{-\lambda_{2}^{k} t}\right]^{m_{k}-l_{k}}\left[e^{-\lambda_{2}^{k} t}\right]^{l_{k}}$

To illustrate the method presented in this section, the lower and upper 215 bounds of survival function for the system in Fig. 2 are calculated. The system has six components belong to two types. Results of survival signature of the system can be seen in Table 1. The failure times of the two component types are according to exponential distribution, with interval parameters $\lambda_{1} \in[0.4,1.2]$ and $\lambda_{2} \in[1.3,2.1]$, respectively. 
Table 1: Survival signature of the system in Fig.2

\begin{tabular}{lll}
\hline$l_{1}$ & $l_{2}$ & $\Phi\left(l_{1}, l_{2}\right)$ \\
\hline 0 & 0 & 0 \\
0 & 1 & 0 \\
0 & 2 & 0 \\
0 & 3 & 0 \\
1 & 0 & 0 \\
1 & 1 & 0 \\
1 & 2 & $1 / 9$ \\
1 & 3 & $1 / 3$ \\
2 & 0 & 0 \\
2 & 1 & 0 \\
2 & 2 & $4 / 9$ \\
2 & 3 & $2 / 3$ \\
3 & 0 & 1 \\
3 & 1 & 1 \\
3 & 2 & 1 \\
3 & 3 & 1 \\
\hline
\end{tabular}




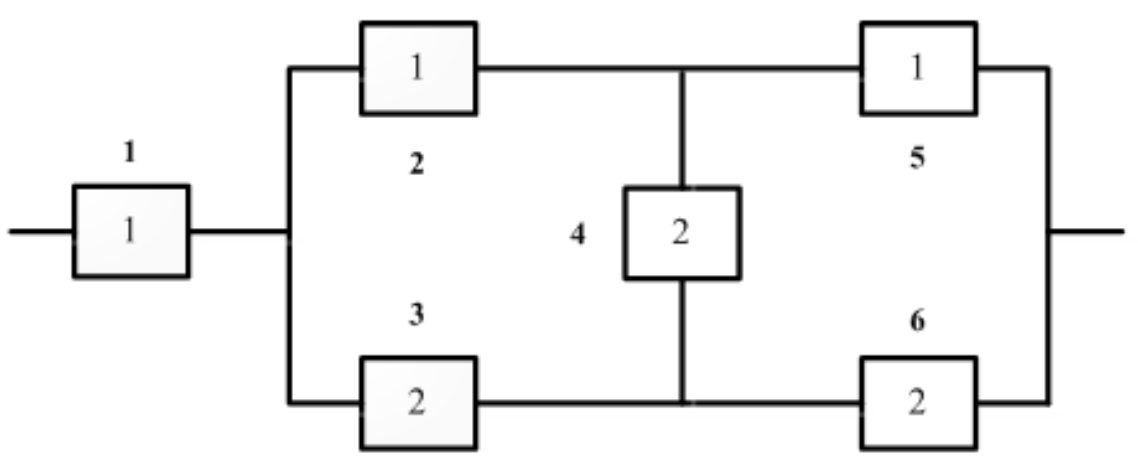

Figure 2: System with two types of components. number of the working components.

The method used to simulate the survival function is derived from the approach proposed in [30]. The simulation approach requires the following steps: 
(1) Sampling the transition times of the first component type, hence a sequence

An important objective of a reliability and risk analysis is to identify those components or events that are most important (critical) from a reliability/safety

(1) for the component type 2, which will obtain 4 additional transition times;

(3) Reordering all the transition times of $\left(t_{1}, t_{2}, \ldots, t_{8}\right)$; (4) For each time interval the probability that the system functions can be computed based on survival signature; (5) Repeating the steps (1) to (4) for $n$ system histories and averaging the obtained results; (6) The system probability of survive over the time $t$ is obtained by averaging the values of survival function.

The above simulation procedures are used for components without imprecision, if there exist imprecision within components failure times, just adding another loop to simulate the components' imprecise parameters. Fig. 3 shows the lower and upper bounds of survival function obtained by simulation method and compared with the analytical solution, and showing a perfect agreement.

The simulation method can be used for analysing any systems with general imprecision. Suppose components failure times of type 1 and type 2 obey Weibull distribution and gamma distribution, respectively. Their imprecise parameters can be seen in Table 2 .

Table 2: Imprecise distribution parameters of components in a system

\begin{tabular}{lll}
\hline Component type & Distribution type & Parameters $(\alpha, \beta)$ \\
\hline 1 & Weibull & $([1.2,1.8],[2.3,2.9])$ \\
2 & Gamma & $([0.8,1.6],[1.3,2.1])$ \\
\hline
\end{tabular}

It is difficult to get the bounds of survival function by analytical method, however, this problem can be tackled through simulation method. The results are shown in Fig. 4.

\section{IMPORTANCE MEASURE OF A SPECIFIC COMPONENT}

\subsection{Definition of Relative Importance Index} components or events that are most important (critical) from a reliability/safety 


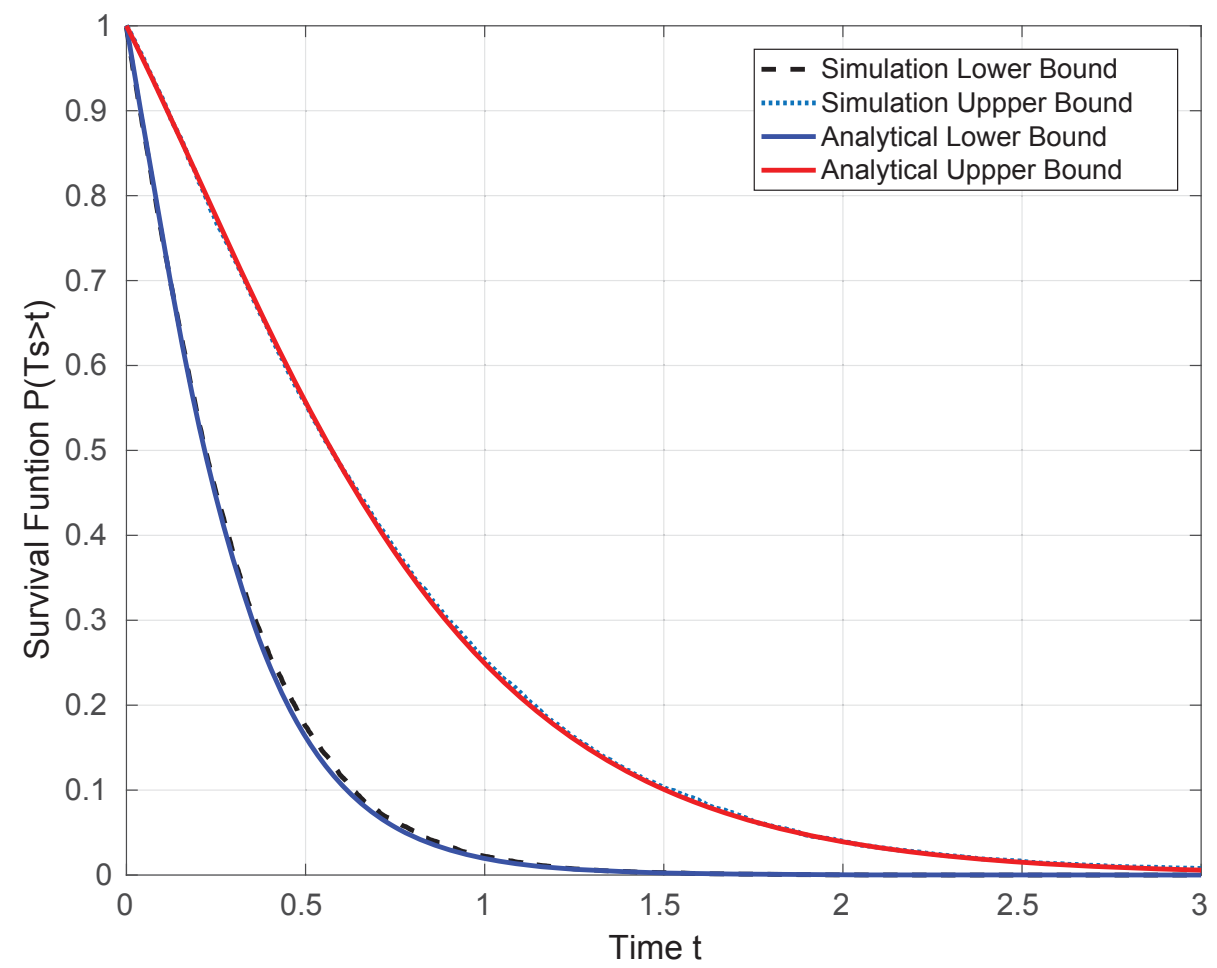

Figure 3: Lower and upper bounds of the survival function obtained by simulation and analytical method.

point of view. These components should be given priority with respect to improvements or maintenance. Importance measures are important tools to evaluate and rank the impact of individual components within a system [31], which will allow one to study the relationship among components and the system. Importance measures have many applications in probabilistic risk analysis and there are many approaches based on various measures of influence and response [32]. These importance measures provide a numerical rank to determine which components are more critical to system failure or more important to system reliability improvement.

A new importance measure is introduced herein as relative importance index indicated by $R I$, which is utilized to quantify the difference between the probability that the system functions if the $i$ th component works and the prob- 


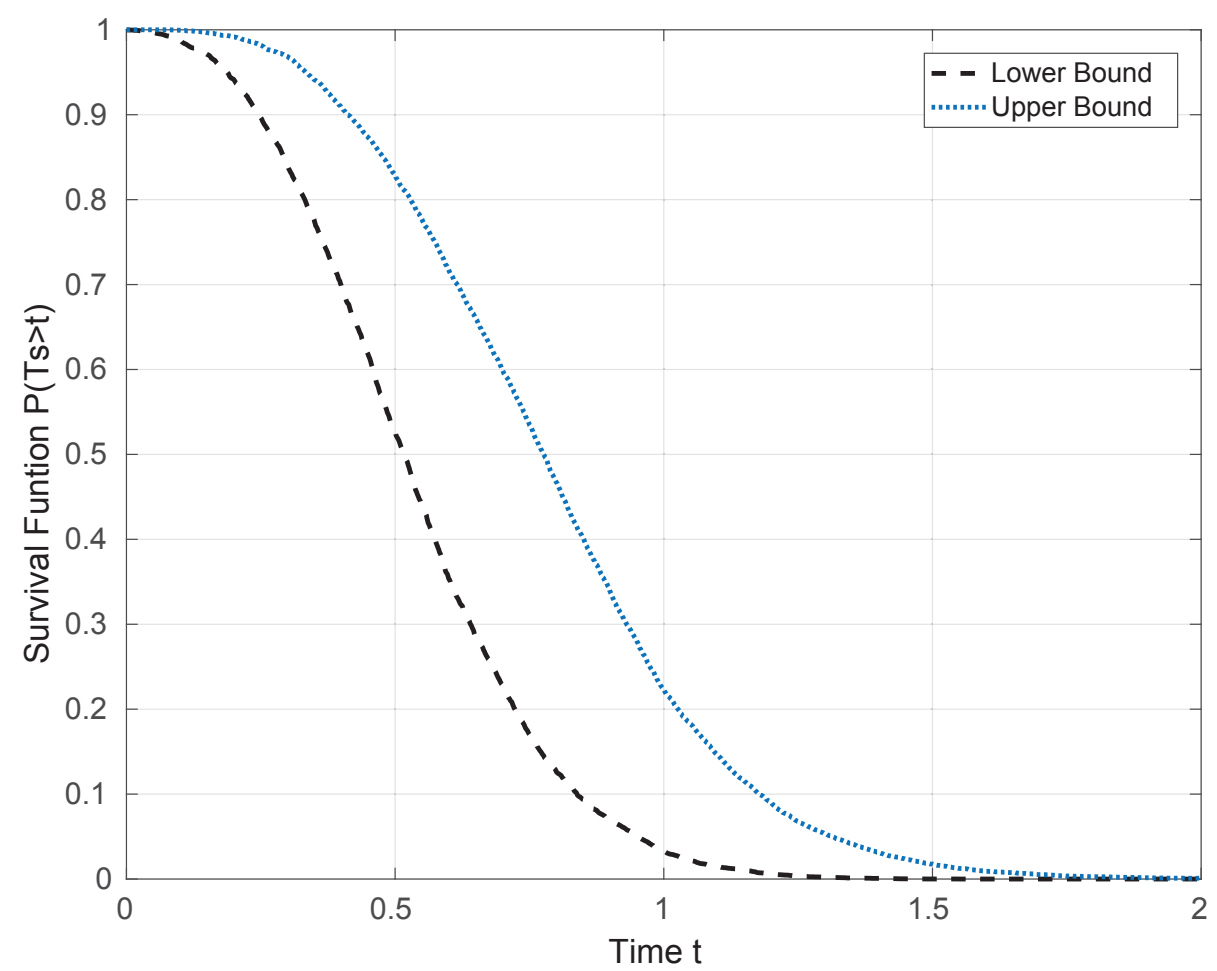

Figure 4: Lower and upper bounds of survival function by simulation method.

ability that the system functions if the $i$ th component is not working. The measure $R I_{i}(t)$ expresses the importance degree of a specific component during the survival time.

The relative importance index $R I_{i}(t)$ can be expressed as follows:

$$
R I_{i}(t)=P\left(T_{S}>t \mid T_{i}>t\right)-P\left(T_{S}>t \mid T_{i} \leq t\right)
$$

Where, $P\left(T_{S}>t \mid T_{i}>t\right)$ represents the probability that the system functions if the $i$ th component works; $P\left(T_{S}>t \mid T_{i} \leq t\right)$ represents the probability that the system functions knowing that the ith component has failed.

The relative importance index $R I_{i}(t)$ is a function of time and it reveals the trend of the survival functions $P\left(T_{S}>t \mid T_{i}>t\right)$ and $P\left(T_{S}>t \mid T_{i} \leq t\right)$ of the system. This measure quantifies the degree of the influence of imprecision in 
Taking imprecise probabilistic characterizations of the component failure probabilities into account, the set of all possible probability distribution functions can be represented as distributional p-boxes [28] indicated with $M: P \in$ $M$. The the relative importance index can be defined as:

$$
R I_{i}(t \mid P)=P\left(T_{S}>t \mid T_{i}>t\right)-P\left(T_{S}>t\left\lceil T_{i} \leq t\right)\right.
$$

Therefore, the lower and upper bounds of relative importance index are:

$$
\begin{gathered}
\underline{R I_{i}}(t)={ }_{P \in M}^{i n f} R I_{i}(t \mid P) \\
\overline{R I_{i}}(t)={ }_{P \in M}^{\text {sup }} R I_{i}(t \mid P)
\end{gathered}
$$

\subsection{Illustrative Example}

Now let calculate the relative importance index of component 4 of the system in section 3.2. First calculate the survival signature of the system in Fig. 5 and Fig. 6, which represents the component 4 of type 2 works and fails at time $t$ respectively.

The survival signature of the two circumstances can be expressed as $\widetilde{\Phi_{1}}\left(l_{1}, l_{2}\right)$ and $\widetilde{\Phi_{0}}\left(l_{1}, l_{2}\right)$, and the results can be seen in Table 3 and Table 4 respectively. So: 
Table 3: Survival signature of the system in Fig.5

\begin{tabular}{lll}
\hline$l_{1}$ & $l_{2}$ & $\Phi\left(l_{1}, l_{2}\right)$ \\
\hline 0 & 0 & 0 \\
0 & 1 & 0 \\
0 & 2 & 0 \\
1 & 0 & 0 \\
1 & 1 & 0 \\
1 & 2 & $1 / 3$ \\
2 & 0 & 0 \\
2 & 1 & $1 / 3$ \\
2 & 2 & $2 / 3$ \\
3 & 0 & 1 \\
3 & 1 & 1 \\
3 & 2 & 1 \\
\hline
\end{tabular}

Table 4: Survival signature of the system in Fig.6

\begin{tabular}{lll}
\hline$l_{1}$ & $l_{2}$ & $\Phi\left(l_{1}, l_{2}\right)$ \\
\hline 0 & 0 & 0 \\
0 & 1 & 0 \\
0 & 2 & 0 \\
1 & 0 & 0 \\
1 & 1 & 0 \\
1 & 2 & $1 / 3$ \\
2 & 0 & 0 \\
2 & 1 & 0 \\
2 & 2 & $2 / 3$ \\
3 & 0 & 1 \\
3 & 1 & 1 \\
3 & 2 & 1 \\
\hline
\end{tabular}




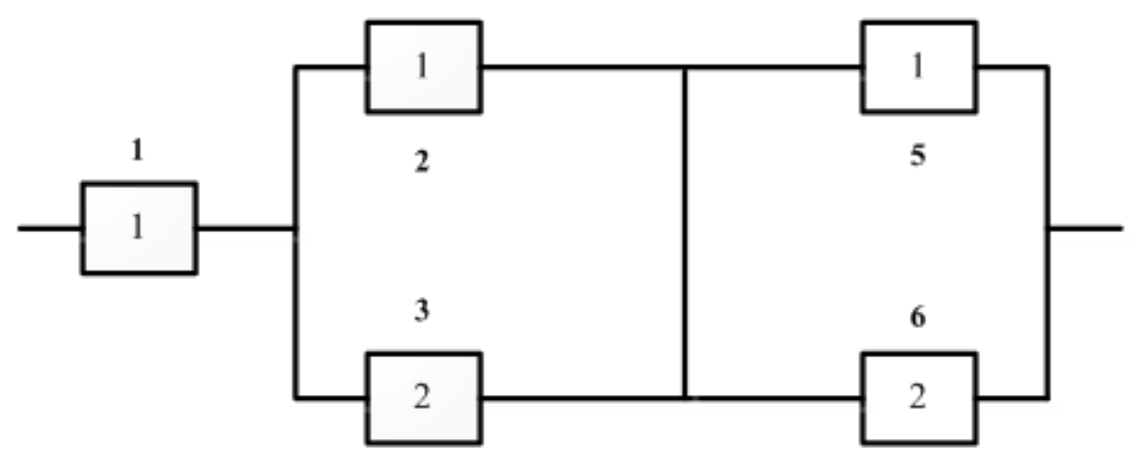

Figure 5: Component 4 works at time $t$.

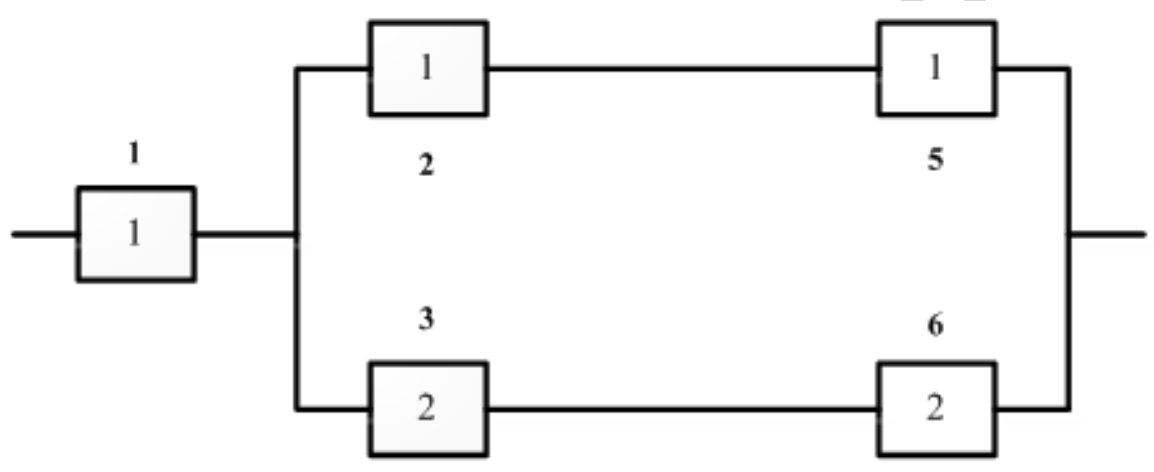

Figure 6: Component 4 fails at time $t$.

$$
\begin{array}{r}
R I_{i}(t \mid P)=P\left(T_{S}>t \mid T_{i}>t\right)-P\left(T_{S}>t \mid T_{i} \leq t\right) \\
=\sum_{l_{1}=0}^{m_{1}} \sum_{l_{2}=0}^{m_{2}-1} \widetilde{\Phi_{1}}\left(l_{1}, l_{2}\right) P\left(\bigcap_{k=1}^{2}\left\{C_{k}(t)=l_{k}\right\}\right)-\sum_{l_{1}=0}^{m_{1}} \sum_{l_{2}=0}^{m_{2}-1} \widetilde{\Phi_{0}}\left(l_{1}, l_{2}\right) P\left(\bigcap_{k=1}^{2}\left\{C_{k}(t)=l_{k}\right\}\right) \\
=\sum_{l_{1}=0}^{m_{1}} \sum_{l_{2}=0}^{m_{2}-1}\left[\widetilde{\Phi_{1}}\left(l_{1}, l_{2}\right)-\widetilde{\Phi_{0}}\left(l_{1}, l_{2}\right)\right] P\left(\bigcap_{k=1}^{2}\left\{C_{k}(t)=l_{k}\right\}\right)
\end{array}
$$

If the components failure times have precise distribution parameters, e.g. 300

$\lambda_{1}=0.8$ and $\lambda_{2}=1.6, M$ degenerates to a probability function $P \equiv M=\{1-$ $\left.e^{-\lambda t}: \lambda_{1}=0.8 ; \lambda_{2}=1.6\right\}$. Hence, the relative importance index of component 
4 can be calculated by using analytical method and the results can be seen in Fig. 7.

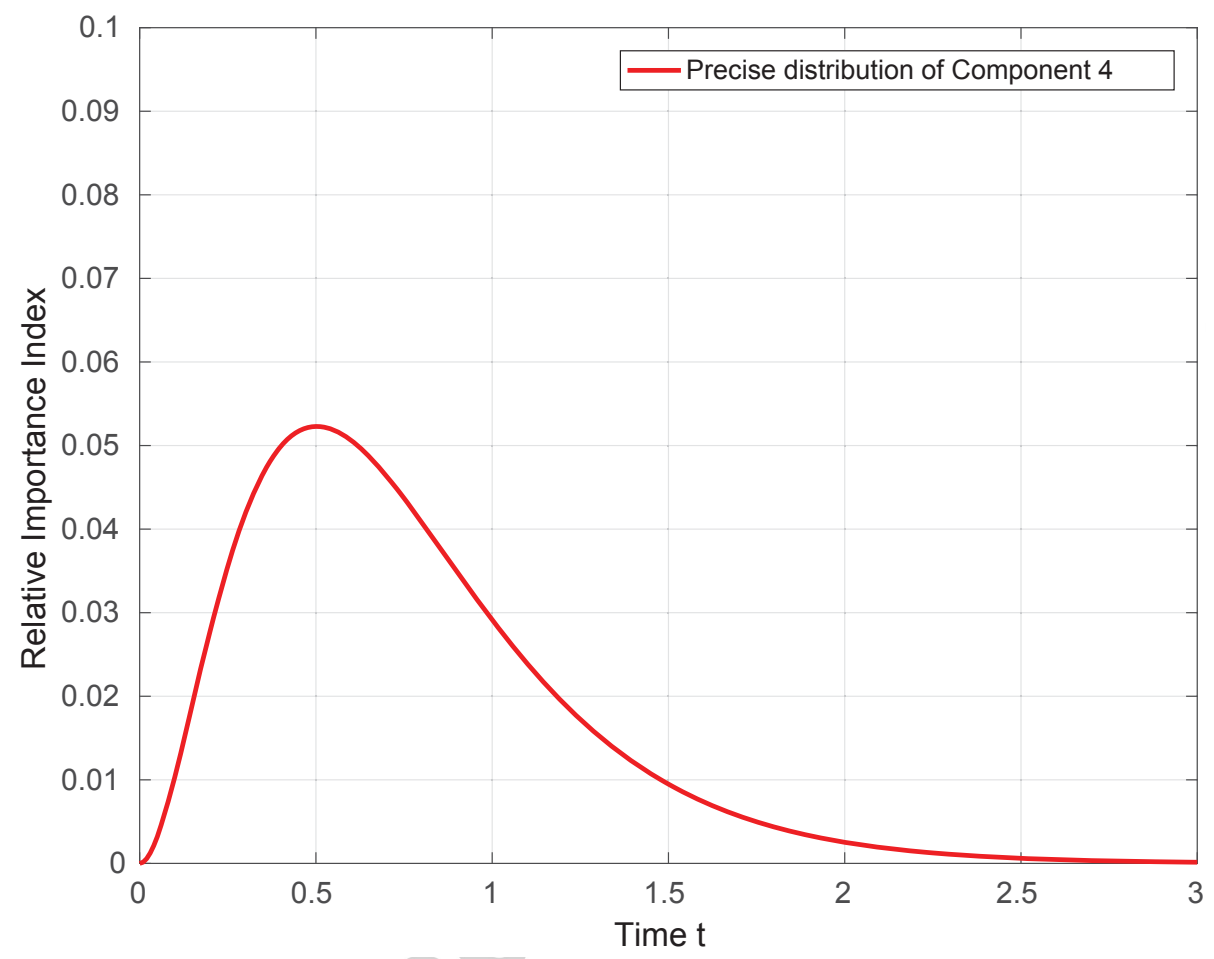

Figure 7: Relative importance index of Component 4 with precise distribution parameters.

Considering imprecisions within components failure times, the set of all prob-

305

$M=\left\{1-e^{-\lambda t}: 0.4 \leq \lambda_{1} \leq 1.2 ; 1.3 \leq \lambda_{2} \leq 2.1\right\}$. Therefore, the lower and upper bounds of relative importance index of component 4 can be calculated through simulation method. Fig. 8 shows the results.

\section{NUMERICAL EXAMPLE}

In this section, a survival analysis of a real world hydro power plant based on survival signature is conducted. The system is schematically shown in Fig. 9 and its reliability block diagram is illustrated in Fig. 10. It can be modelled as 


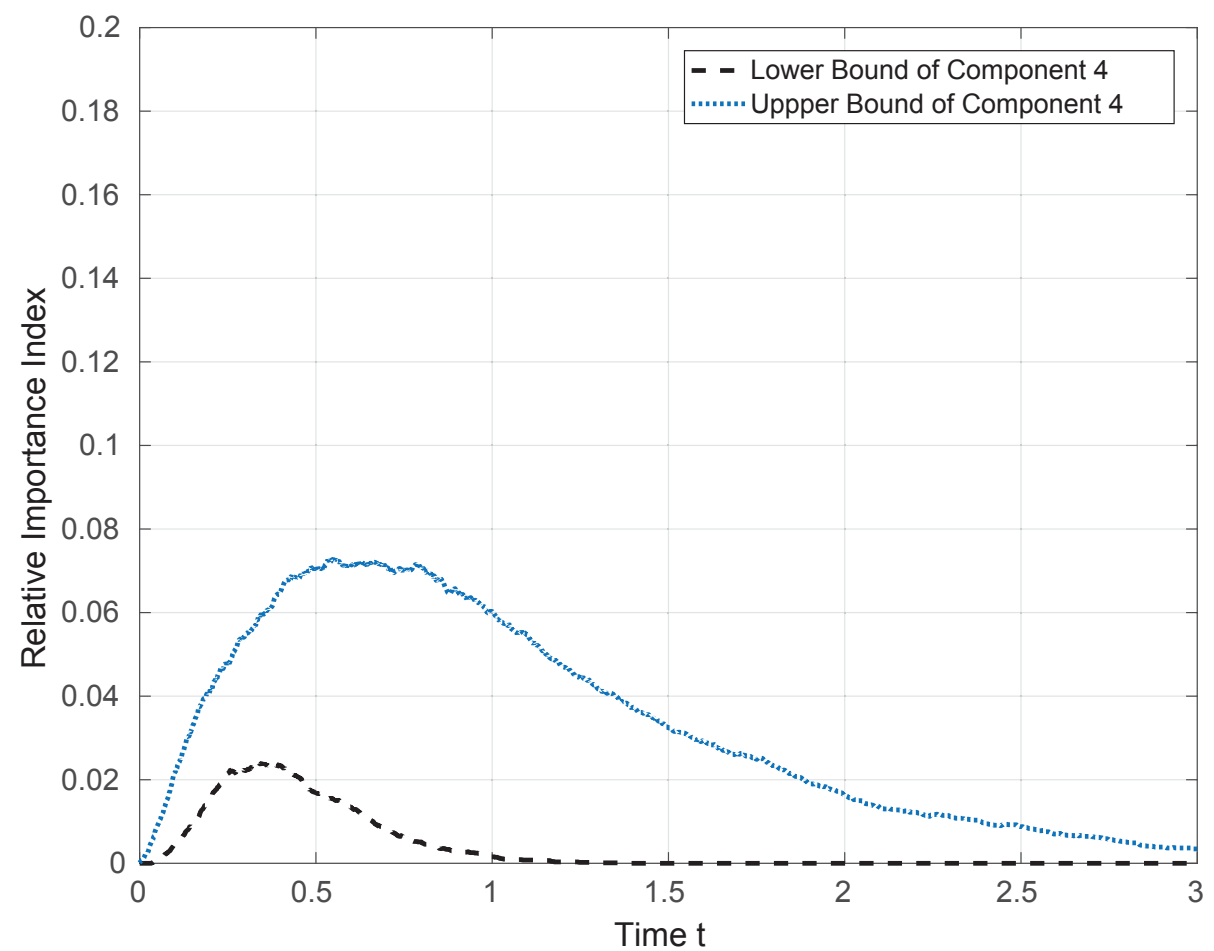

Figure 8: Relative importance index of Component 4 with imprecise distribution parameters.

a complex system comprising the following main twelve components: (1) control gate $(C G)$, which is built on the inside of the dam, the water from the reservoir is released and controlled through the gate; (2) two butterfly valves $(B V 1, B V 2)$, which can transport and control the water flow; (3) two turbines $(T 1, T 2)$, where the flowing waters kinetic energy is transformed into mechanical energy; (4) three circuit breakers $(C B 1, C B 2, C B 3)$, which are used to protect the hydro power plant system; (5) two generators $(G 1, G 2)$, which produce alternating current by moving electrons; and (6) two transformers ( $T X 1, T X 2)$, which inside the powerhouse take the alternating current and convert it to higher-voltage current.

Two cases are presented in the following part: Case A presents the survival analysis with the fully probability model; Case B considers imprecision within 325 the model. 


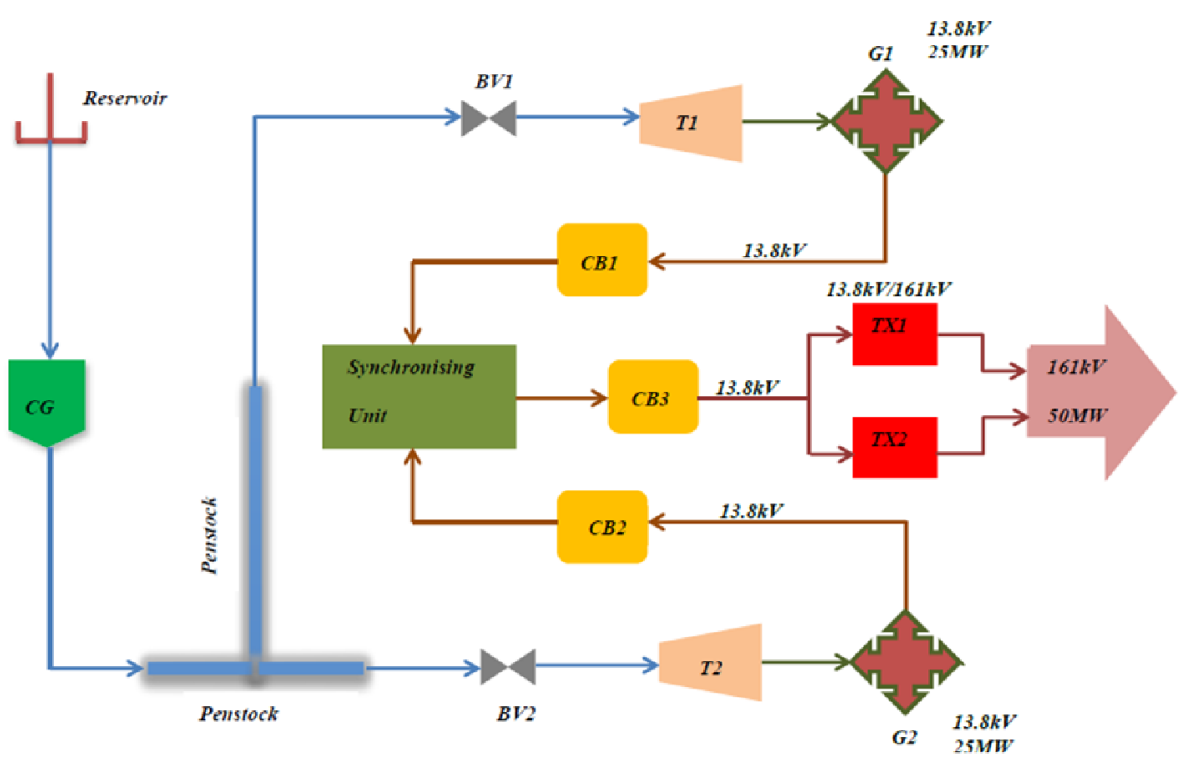

Figure 9: Schematic diagram of a hydro power plant system.

\subsection{Case A}

It is assumed that all components of the same type have the same failure time distribution. Failure type and distribution parameters are listed in Table 5.

Let $l_{1}, l_{2}, l_{3}, l_{4}, l_{5}$ and $l_{6}$ denote $C G, B V, T, G, C B$ and $T X$, respectively. Table 6 shows the survival signature of the hydro power plant, whereby the rows with values $\Phi\left(l_{1}, l_{2}, l_{3}, l_{4}, l_{5}, l_{6}\right)=0$ are omitted.

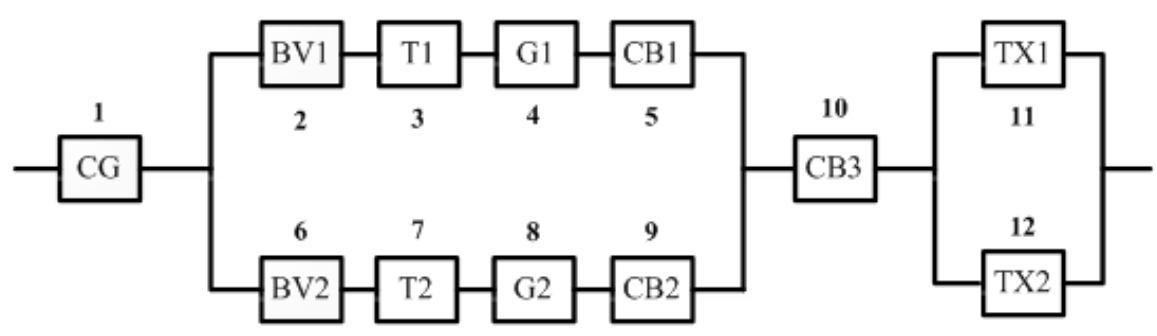

Figure 10: Reliability block diagram of a hydro power plant system. 
Table 5: Failure types and distribution parameters of components in a hydro power plant

\begin{tabular}{lll}
\hline Component name & Distribution type & Parameters $(\alpha, \beta)$ or $\lambda$ \\
\hline$C G$ & Weibull & $(1.3,1.8)$ \\
$B V$ & Weibull & $(1.2,2.3)$ \\
$T$ & Exponential & 0.8 \\
$G$ & Weibull & $(1.6,2.6)$ \\
$C B$ & Gamma & $(1.3,3.0)$ \\
$T X$ & Gamma & $(0.6,1.1)$ \\
\hline
\end{tabular}

Table 6: Survival signature of a hydro power plant in Fig.9; rows with $\Phi\left(l_{1}, l_{2}, l_{3}, l_{4}, l_{5}, l_{6}\right)=0$

\begin{tabular}{lllllll}
\hline \begin{tabular}{llll} 
are omitted \\
\hline$l_{1}$
\end{tabular} & $l_{2}$ & $l_{3}$ & $l_{4}$ & $l_{5}$ & $l_{6}$ & $\Phi\left(l_{1}, l_{2}, l_{3}, l_{4}, l_{5}, l_{6}\right)$ \\
\hline 1 & 1 & 1 & 1 & 2 & {$[1,2]$} & $1 / 12$ \\
1 & 1 & 1 & 2 & 2 & {$[1,2]$} & $1 / 6$ \\
1 & 1 & 2 & 1 & 2 & {$[1,2]$} & $1 / 6$ \\
1 & 2 & 1 & 1 & 2 & {$[1,2]$} & $1 / 6$ \\
1 & 1 & 1 & 1 & 3 & {$[1,2]$} & $1 / 4$ \\
1 & 1 & 2 & 2 & 2 & {$[1,2]$} & $1 / 3$ \\
1 & 2 & 1 & 2 & 2 & {$[1,2]$} & $1 / 3$ \\
1 & 2 & 2 & 1 & 2 & {$[1,2]$} & $1 / 3$ \\
1 & 1 & 1 & 2 & 3 & {$[1,2]$} & $1 / 2$ \\
1 & 1 & 2 & 1 & 3 & {$[1,2]$} & $1 / 2$ \\
1 & 2 & 1 & 1 & 3 & {$[1,2]$} & $1 / 2$ \\
1 & 2 & 2 & 2 & 2 & {$[1,2]$} & $2 / 3$ \\
1 & 1 & 2 & 2 & 3 & {$[1,2]$} & 1 \\
1 & 2 & 1 & 2 & 3 & {$[1,2]$} & 1 \\
1 & 2 & 2 & {$[1,2]$} & 3 & {$[1,2]$} & 1 \\
\hline
\end{tabular}


The survival signature can now be used as follows. There are $m_{1}=1, m_{2}=$ $m_{3}=m_{4}=m_{6}=2$ and $m_{5}=3$ components of each type. The survival signature must consider combinations for all $l_{1} \in\{0,1\}, l_{2}, l_{3}, l_{4}, l_{6} \in\{0,1,2\}$ and $l_{5} \in$ $\{0,1,2,3\}$, and the state vector is $\underline{x}=\left(x_{1}^{1}, x_{1}^{2}, x_{2}^{2}, x_{1}^{3}, x_{2}^{3}, x_{1}^{4}, x_{2}^{4}, x_{1}^{5}, x_{2}^{5}, x_{3}^{5}, x_{1}^{6}, x_{2}^{6}\right)$. Now consider $\Phi(1,1,1,2,2,1)$ for example. This covers all possible vectors $\underline{x}$ with $x_{1}^{1}=1, x_{1}^{2}+x_{2}^{2}=1, x_{1}^{3}+x_{2}^{3}=1, x_{1}^{4}+x_{2}^{4}=2, x_{1}^{5}+x_{2}^{5}+x_{3}^{5}=2$ and $x_{1}^{6}+x_{2}^{6}=1$. There are 24 such vectors, but only four of these can make the system function. Due to the iid assumption of the failure times of components of the same type, and due to independence between components of different types, all these 24 vectors have equal probability to occur, hence $\Phi(1,1,1,2,2,1)=4 / 24=1 / 6$.

The survival function of the hydro power plant system with twelve components of six types is shown in Fig. 11.

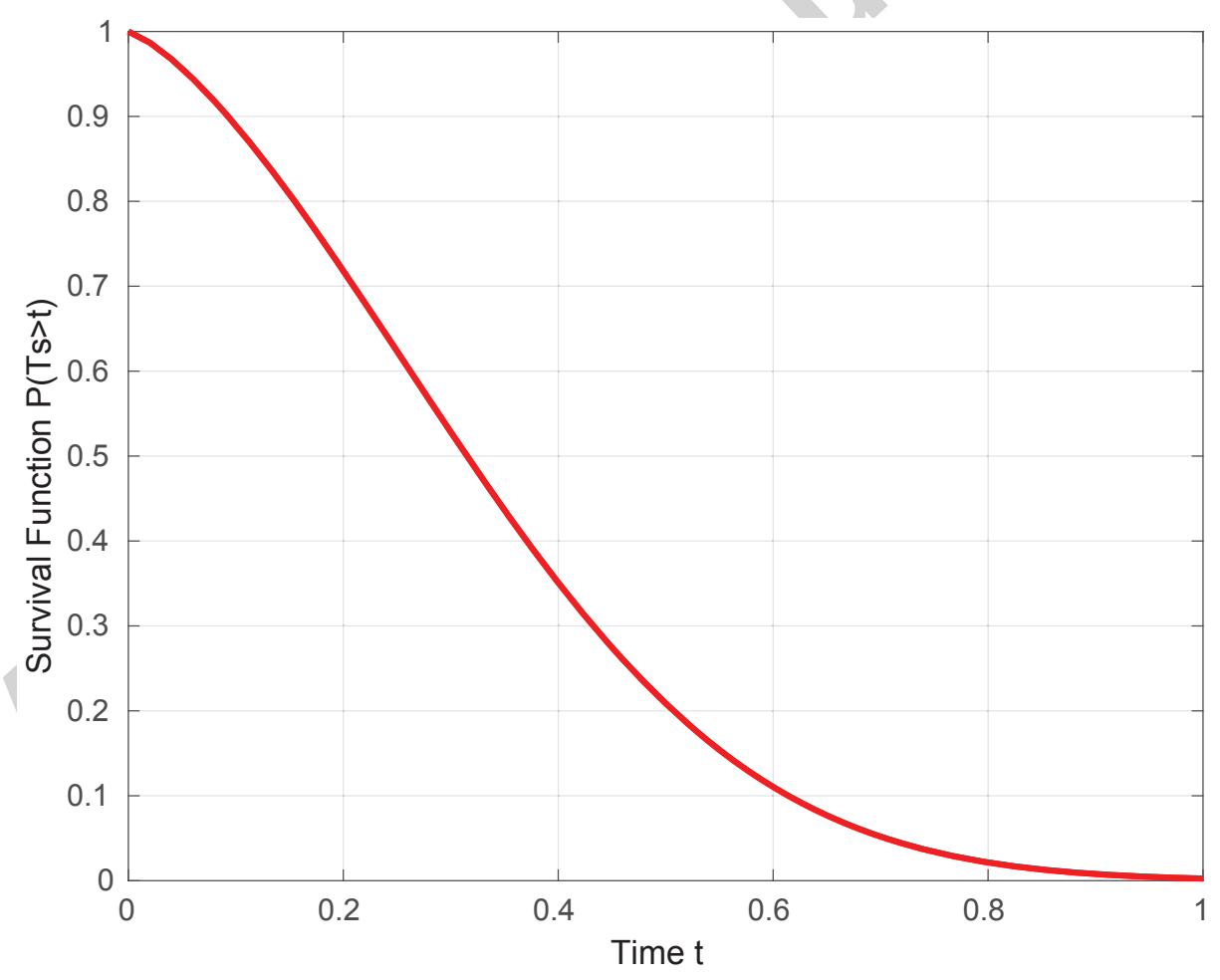

Figure 11: Survival function of a hydro power plant system along with survival functions for the individual components. 
Based on the survival function it is possible to calculate the influence of each component on the system reliability for each point in time $t$. The basic theoretical knowledge and equations can be seen in Section 4, which allows to estimate of relative importance index $R I_{i}(t)$ of each component.

For the other component importance measures, analytical methods can be used to rank the component importance degree. The equations of Birnbaum's measure $(B M)$, risk achievement worth $(R A W)$ and Fussel-Vesely's measure $(F V)$ to calculate the component importance $I_{i}(t)$ of the $i$ th component at time $t$ can be seen in Table 7 .

Table 7: Component importance equations of $B M, R A W$ and $F V$

\begin{tabular}{ll}
\hline Methods & Component Importance Equations \\
\hline$B M$ & $I_{i}^{B}(t)=\frac{\partial R_{S}(t)}{\partial R_{i}(t)}$ \\
$R A W$ & $I_{i}^{R A W}(t)=\frac{R_{S}(t)\left(R_{i}(t)=1\right)}{R_{S}(t)}$ \\
$F V$ & $I_{i}^{F V}(t)=\frac{R_{S}(t)-R_{S}(t)\left(R_{i}(t)=0\right)}{R_{S}(t)}$ \\
\hline
\end{tabular}

In the above equations, $R_{S}(t)$ and $R_{i}(t)$ represent the reliability of the system and the $i$ th component at time $t$. For the power plant in Fig. 9, the reliability equation $R_{S}(t)=R_{1}\left(1-\left(1-R_{2} R_{3} R_{4} R_{5}\right)\left(1-R_{6} R_{7} R_{8} R_{9}\right)\right) R_{10}\left(1-\left(1-R_{11}\right)(1-\right.$ $\left.R_{12}\right)$ ).

The component importance obtained at $t=0.12$ using the proposed method for the power plant system have been compared with the results Birnbaum's measure $(B M)$, risk achievement worth $(R A W)$ and Fussel-Vesely's measure $(F V)$ as shown in Table 8.

According to the above table, it can be drawn that $R I$ method can get the same component importance ranking as Birnbaum's measure. Also, the proposed $R I$ method has the same ranking trend as $R A W$ and $F V$. The $R I$ method just needs the survival signature without calculating the reliability equation, which is useful for large systems with multiple component types.

The relative importance index values of each components over the time are shown in Fig. 12. 


\begin{tabular}{llllllll}
\multicolumn{6}{c}{ Table 8: Comparision of component importance obtained using different methods at $t=0.12$} \\
Components & $C G$ & $B V 1$ & $T 1$ & $G 1$ & $C B 1$ & $C B 3$ & $T X 1$ \\
Methods & & $B V 2$ & $T 2$ & $G 2$ & $C B 2$ & & $T X 2$ \\
\hline$B M$ & 0.8854 & 0.1181 & 0.1366 & 0.1177 & 0.1191 & 0.8846 & 0.2703 \\
ranking & 1 & 6 & 4 & 7 & 5 & 2 & 3 \\
\hline$R A W$ & 7.8947 & 1.9280 & 1.9280 & 1.9280 & 1.9280 & 7.8947 & 2.5270 \\
ranking & 1 & 3 & 3 & 3 & 3 & 1 & 2 \\
\hline$F V$ & 1.000 & 0.1346 & 0.1346 & 0.1346 & 0.1346 & 1.000 & 0.2215 \\
ranking & 1 & 3 & 3 & 3 & 3 & 1 & 2 \\
\hline$R I$ & 0.8831 & 0.1217 & 0.1401 & 0.1213 & 0.1221 & 0.8693 & 0.2656 \\
ranking & 1 & 6 & 4 & 7 & 5 & 2 & 3 \\
\hline
\end{tabular}

The relative importance index values reveal the component importance over time. The bigger the value of $R I_{i}(t)$ is, the more "critical" the $i$ th component is. The above results show that $B V 1$ and $B V 2$ have the same relative importance index values, and the same applies to $T 1$ and $T 2, G 1$ and $G 2, C B 1$ and $C B 2$, $T X 1$ and $T X 2$. This is because the components are in a parallel configuration and they have the same failure time distribution type and parameters, which is also according to our common sense that these components have the same importance degree to the system. For component $C B 3$, it has same failure time type and distribution parameters as components $C B 1$ and $C B 2$, but has different location in the system. Therefore, the relative importance index value of component $C B 3$ is bigger than relative importance index values of components $C B 1$ and $C B 2$, but not as big as the relative importance index value of component $C G$. Components $C G$ and $C B 3$ have the same decreasing trend of relative importance index over time, while for the other components, the trends of relative importance index increase first, then decay with time. The relative importance index values of components $T X 1$ and $T X 2$ are always smaller than other components, which means they have smallest influence degree to the system reliability. 


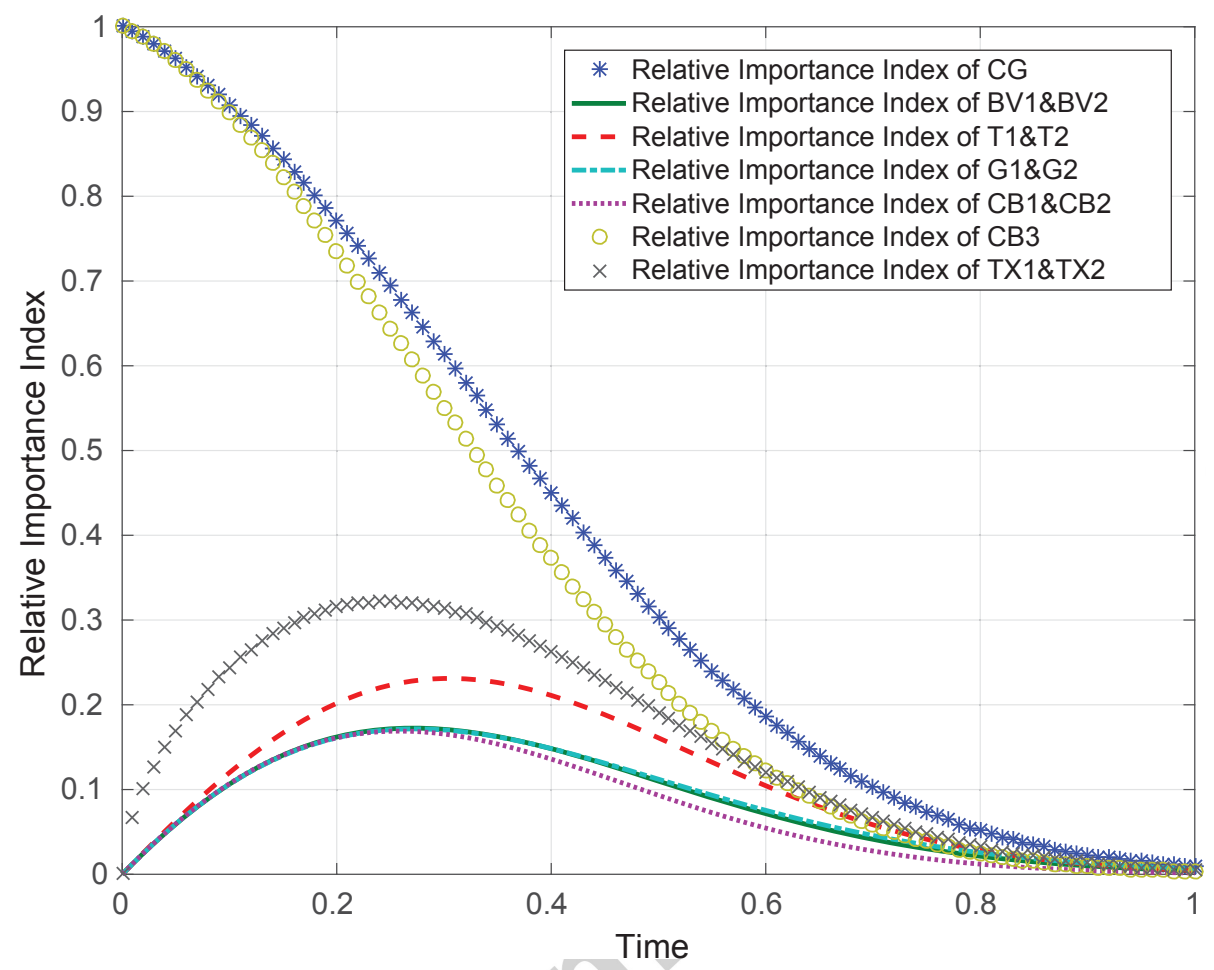

Figure 12: Relative importance index values of the system components.

\subsection{Case $B$}

The investigation from CASE A is now extended by considering imprecision in the description of the probabilistic model for the failure characterization of the system components. Intervals are used to describe the imprecision in the failure time distribution as shown in Table 9.

The upper and lower bounds of the parameters reflect the ideal and the worst case of the performance of the components, respectively. The range of the parameters represents epistemic uncertainty, which results from expert assessments of the component performance. This modelling leads to upper and lower survival functions of the hydro power plant system reflecting the epistemic uncertainties as range between the curves, see Fig. 13. The imprecision from the input is translated into imprecision of the output.

As a further step the imprecision can be carried forward to calculate ranges 


\begin{tabular}{lll}
\multicolumn{3}{l}{ Table 9: Failure types and distribution parameters of components in a hydro power plant } \\
\hline Component name & Distribution type & Parameters $(\alpha, \beta)$ or $\lambda$ \\
\hline$C G$ & Weibull & $([1.2,1.5],[1.5,2.1])$ \\
$B V$ & Weibull & $([1.0,1.6],[2.1,2.5])$ \\
$T$ & Exponential & {$[0.4,1.2]$} \\
$G$ & Weibull & $([1.3,1.8],[2.3,2.9])$ \\
$C B$ & Gamma & $([1.2,1.4],[2.8,3.3])$ \\
$T X$ & Gamma & $([0.3,0.8],[1.0,1.3])$ \\
\hline
\end{tabular}

400

for the relative importance index. Firstly, ranges for the survival functions assuming given component fails or works are calculated for each component, then the associated ranges for the relative importance index for each component are determined, see Fig. 14 and Fig. 15.

From the above figures it can be recognized that imprecision within component failure times can lead to imprecision of relative importance index of the component.

\section{CONCLUSIONS}

In this paper an efficient approach for analysing imprecise system reliability and component importance has been presented. The method is based on the survival signature, which has been proven to be an effective method to estimate the survival function of systems with multiple component types. In the proposed approach, the system model needs to be analysed only once in order to conduct a reliability analysis and measure a component importance, which represents a significant computational advantage. Performing a survival analysis on systems using the survival signature has been presented as a novel pathway for system reliability and component importance. In addition, the effect of imprecision, for example resulting from incomplete data, has been taken into account in the system reliability analysis and component importance measurement. As a consequence, bounds of survival functions of the system and intervals of relative 


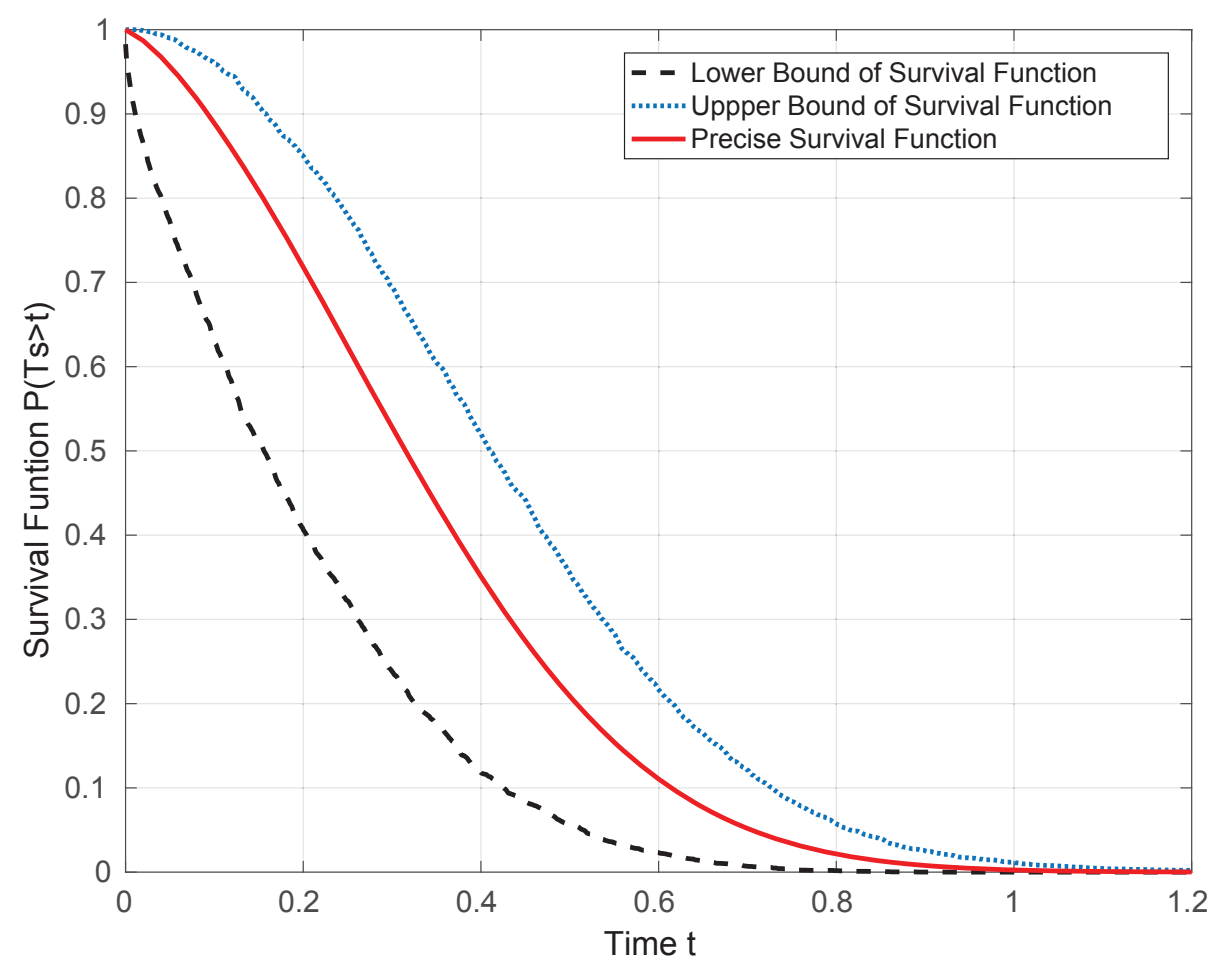

Figure 13: Upper, lower and precise survival functions of the hydro power plant system.

420

importance index values can be obtained.

In order to quantify the influence degree of components without and with imprecision, a novel component-wise importance measure has been presented: the relative importance index. Importance measures allow to identify the most "critical" system component at a specific time. This allows an optimal allocation of resources for repair, maintenance and inspection. This novel and efficient method is conducted in an analytical way or through simulation method based on survival signature, which improves the computational efficiency. Using the relative importance index, the importance of the individual components is ranked to obtain a preference list for maintenance and repair. The effectiveness and feasibility of the proposed approaches have been demonstrated with some numerical examples. The results show that the survival signature is an efficient method to perform a reliability analysis of systems and measure components 


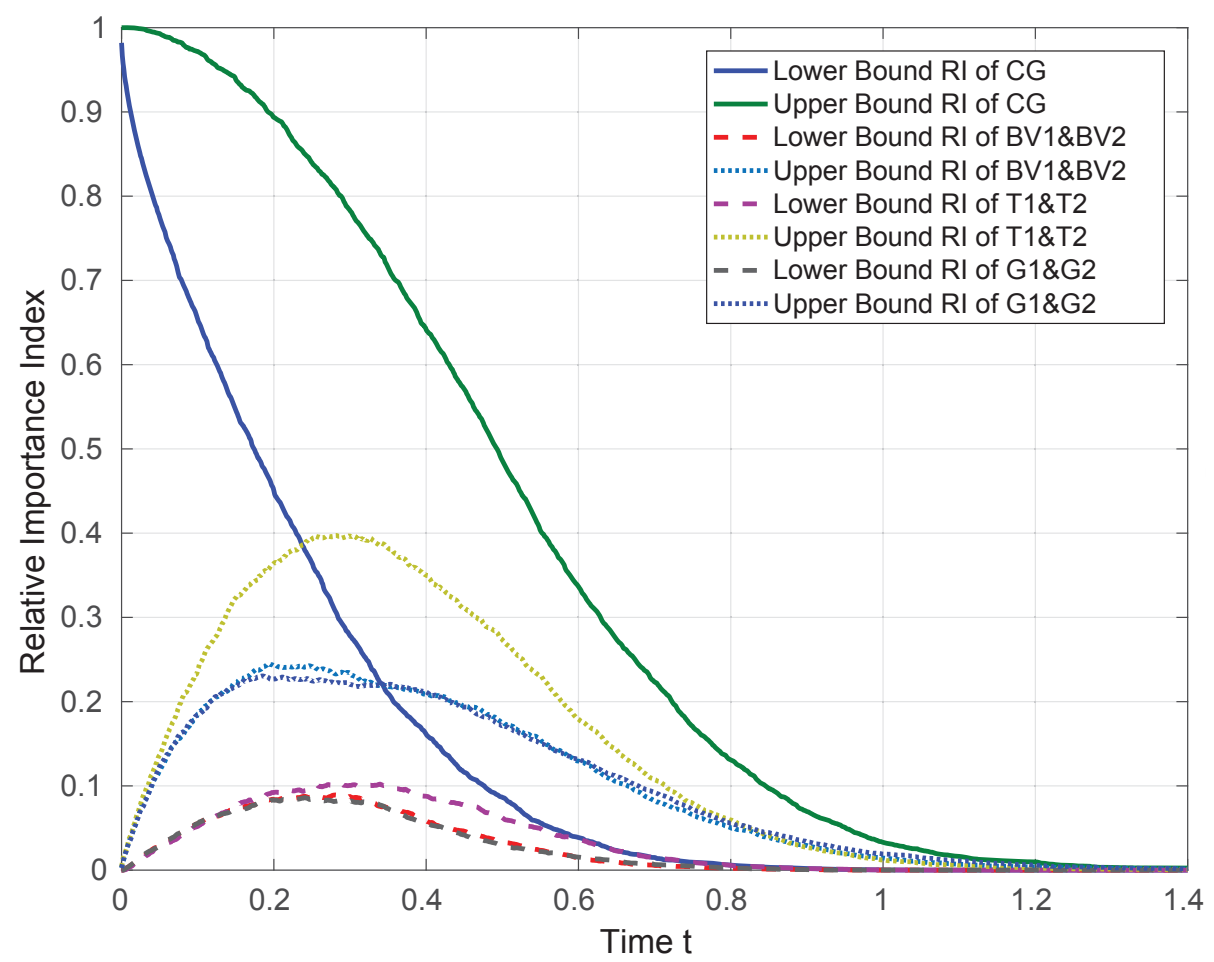

Figure 14: Upper and lower relative importance index of components $C G, B V, T$ and $G$.

importance.

\section{References}

[1] P. R. Adduri, R. C. Penmetsa, System reliability analysis for mixed uncertain variables, Structural Safety 31 (5) (2009) 375-382.

[2] R. G. Miller Jr, Survival analysis, Vol. 66, John Wiley \& Sons, 2011.

[3] F. J. Samaniego, System signatures and their applications in engineering reliability, Vol. 110, Springer Science \& Business Media, 2007.

440

[4] S. Eryilmaz, Review of recent advances in reliability of consecutive k-outof-n and related systems, Proceedings of the Institution of Mechanical Engineers, Part O: Journal of Risk and Reliability 224 (3) (2010) 225-237. 


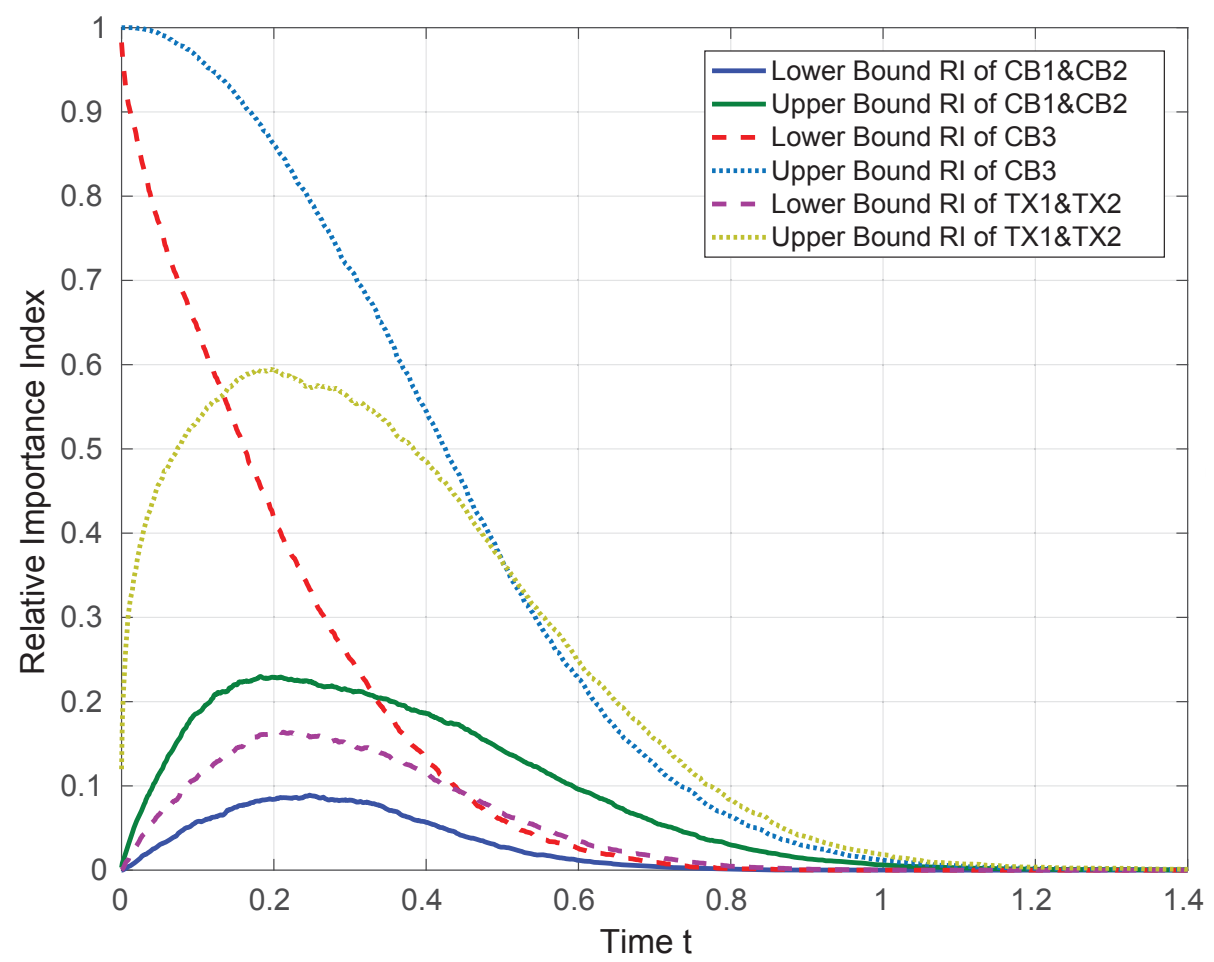

Figure 15: Upper and lower relative importance index of components $C B$ and $T X$.

[5] F. P. Coolen, T. Coolen-Maturi, Generalizing the signature to systems with multiple types of components, in: Complex Systems and Dependability, Springer, 2012, pp. 115-130.

[6] F. P. Coolen, T. Coolen-Maturi, Modelling uncertain aspects of system dependability with survival signatures, in: Dependability Problems of Complex Information Systems, Springer, 2015, pp. 19-34.

[7] L. J. Aslett, F. P. Coolen, S. P. Wilson, Bayesian inference for reliability of systems and networks using the survival signature, Risk Analysis 35 (3) (2015) 1640-1651.

[8] Z. W. Birnbaum, On the importance of different components in a multicomponent system, Tech. rep., DTIC Document (1968). 
[9] A. Høyland, M. Rausand, System reliability theory: models and statistical methods, Vol. 420, John Wiley \& Sons, 2009.

[10] G. Levitin, A. Lisnianski, Importance and sensitivity analysis of multistate systems using the universal generating function method, Reliability Engineering \& System Safety 65 (3) (1999) 271-282.

[11] J. Fussell, How to hand-calculate system reliability and safety characteristics, Reliability, IEEE Transactions on 24 (3) (1975) 169-174.

[12] M. J. Armstrong, Reliability-importance and dual failure-mode components, Reliability, IEEE Transactions on 46 (2) (1997) 212-221.

[13] A. Gandini, Importance and sensitivity analysis in assessing system reliability, Reliability, IEEE Transactions on 39 (1) (1990) 61-70.

[14] W. Wang, J. Loman, P. Vassiliou, Reliability importance of components in a complex system, in: Reliability and Maintainability, 2004 Annual Symposium-RAMS, IEEE, 2004, pp. 6-11.

[15] E. Zio, L. Podofillini, Monte carlo simulation analysis of the effects of different system performance levels on the importance of multi-state components, Reliability Engineering \& System Safety 82 (1) (2003) 63-73.

[16] E. Zio, L. Podofillini, G. Levitin, Estimation of the importance measures of multi-state elements by monte carlo simulation, Reliability Engineering \& System Safety 86 (3) (2004) 191-204.

[17] M. Beer, S. Ferson, V. Kreinovich, Imprecise probabilities in engineering analyses, Mechanical systems and signal processing 37 (1) (2013) 4-29.

[18] M. Beer, Y. Zhang, S. T. Quek, K. K. Phoon, Reliability analysis with scarce information: Comparing alternative approaches in a geotechnical engineering context, Structural Safety 41 (2013) 1-10.

[19] M. Beer, V. Kreinovich, Interval or moments: which carry more information?, Soft Computing 17 (8) (2013) 1319-1327. 
[20] B. Möller, M. Beer, Engineering computation under uncertaintycapabilities of non-traditional models, Computers \& Structures 86 (10) (2008) 1024-1041.

[21] E. Patelli, M. Broggi, M. d. Angelis, M. Beer, Opencossan: An efficient open tool for dealing with epistemic and aleatory uncertainties, in: Vulnerability, Uncertainty, and Risk Quantification, Mitigation, and Management, ASCE, 2014, pp. 2564-2573.

[22] E. Patelli, H. J. Pradlwarter, G. I. Schuëller, Global sensitivity of structural variability by random sampling, Computer Physics Communications 181 (12) (2010) 2072-2081.

[23] E. Patelli, H. M. Panayirci, M. Broggi, B. Goller, P. Beaurepaire, H. J. Pradlwarter, G. I. Schuëller, General purpose software for efficient uncertainty management of large finite element models, Finite elements in analysis and design 51 (2012) 31-48.

[24] F. P. Coolen, T. Coolen-Maturi, A. H. Al-Nefaiee, Nonparametric predictive inference for system reliability using the survival signature, Proceedings of the Institution of Mechanical Engineers, Part O: Journal of Risk and Reliability 228 (5) (2014) 437-448.

[25] S. Ferson, J. Hajagos, W. T. Tucker, Probability bounds analysis is a global sensitivity analysis, in: International Conference on Sensitivity Analysis of Model Output (SAMO), 2004.

[26] S. Ferson, J. G. Hajagos, Arithmetic with uncertain numbers: rigorous and (often) best possible answers, Reliability Engineering \& System Safety 85 (1) (2004) 135-152.

[27] S. Ferson, C. A. Joslyn, J. C. Helton, W. L. Oberkampf, K. Sentz, Summary from the epistemic uncertainty workshop: consensus amid diversity, Reliability Engineering \& System Safety 85 (1) (2004) 355-369. 
[28] S. Ferson, V. Kreinovich, L. Ginzburg, D. S. Myers, K. Sentz, Constructing probability boxes and Dempster-Shafer structures, Vol. 835, Sandia National Laboratories, 2002.

[29] E. Wolfstetter, et al., Stochastic dominance: theory and applications, Humboldt-Univ., Wirtschaftswiss. Fak., 1993.

[30] E. Zio, P. Baraldi, E. Patelli, Assessment of the availability of an offshore installation by monte carlo simulation, International Journal of Pressure Vessels and Piping 83 (4) (2006) 312-320.

[31] J. E. Ramirez-Marquez, D. W. Coit, Composite importance measures for multi-state systems with multi-state components, Reliability, IEEE Transactions on 54 (3) (2005) 517-529.

[32] E. Patelli, D. A. Alvarez, M. Broggi, M. d. Angelis, Uncertainty management in multidisciplinary design of critical safety systems, Journal of Aerospace Information Systems 12 (1) (2014) 140-169. 